\title{
A special class of fuzzy measures: Choquet integral and applications
}

\author{
Domenico Candeloro $^{a}$, Radko Mesiar ${ }^{b, *}$, Anna Rita Sambucini ${ }^{a}$ \\ ${ }^{a}$ Department of Mathematics and Computer Sciences, University of Perugia, Via Vanvitelli 1, 06123 Perugia (Italy) \\ ${ }^{b}$ Slovak University of Technology (STU) Bratislava Faculty of Civil Engineering, Department of Mathematics \\ Radlinského 11, 81005 Bratislava, (Slovakia)
}

\begin{abstract}
Core of an economy and Walras equilibria are considered for a product space $X \times[0,1]$ using the Choquet integral with respect to a fuzzy measure.

Keywords: fuzzy measure, sectional function, Choquet integral, Walrasian equilibria, Core of an economy

2010 MSC: 28B20, 26E25, 46B20, 54C60
\end{abstract}

\section{Introduction}

The Choquet integral, introduced by G. Choquet in 1953 ([20]), gives a method to integrate functions with respect to non necessarily additive measures such as capacities or, more generally, fuzzy measures $([9-11,14,15,29,30,32-34,37,40])$. Besides its initial applications in potential theory and statistical mechanics it became a useful tool to deal with uncertainty in imprecise probability theory, in decision theory and in the study of cooperative games ([22, 23, 36, 39, 44]). The Choquet integral has applications also in finance, economics and insurance. One of the central problems in Mathematical Economics is the search of equilibria for the model; in the finitely additive framework results are given in $[1-3,5-8,43]$. In this research, we assume that the space of agents is decomposed into a large number of sections, each of which is an authonomous economic model, but coalitions can be created also among members of different sections, according with some rules.

The mathematical model is a product space $X^{*}:=X \times[0,1]$, where the sections are the sets $X \times\{y\}$, as $y$ ranges in $[0,1]$. The set $X$ represents the typical section of agents, and is endowed with a $\sigma$-algebra $\mathcal{A}$, while in the $[0,1]$ space the usual Lebesgue $\sigma$-algebra $\mathcal{B}$ and measure $\lambda$ are fixed. In each section $X \times\{y\}$ the obvious $\sigma$-algebra $\mathcal{A} \times\{y\}$ is considered, with a fuzzy measure $\mu_{y}$ defined there.

The coalitions are all the sets of the product $\sigma$-algebra $\mathcal{H}$ generated by $\mathcal{A}$ and the Borel $\sigma$ algebra $\mathcal{B}$, and the fuzzy measure $m: \mathcal{H} \rightarrow \mathbb{R}_{0}^{+}$is defined by integrating the measures $\mu_{y}$ with

\footnotetext{
* corresponding author address: Slovak University of Technology (STU) Bratislava Faculty of Civil Engineering, Department of Mathematics Radlinského 11, 81005 Bratislava, (Slovakia)

Email addresses: domenico.candeloro@unipg. it (Domenico Candeloro), mesiar@math.sk (Radko Mesiar), anna.sambucini@unipg.it (Anna Rita Sambucini)

Preprint submitted to Elsevier 
respect to the Lebesgue measure in $[0,1]$. This model includes the case in which the sections are just a finite number of sets, $E_{1}, \ldots, E_{r}$, and $\mu$ is additive along them. Indeed, in that case it will suffice to define $X$ as the union of these sets, and to decompose [0,1] into $r$ subintervals $J_{i}$ (such that $\left.\lambda\left(J_{i}\right)=\mu\left(E_{i}\right)\right)$, in each point $y$ of which the measure $\mu_{y}$ is null outside the set $E_{i} \times\{y\}$ and coincides with $\frac{\mu}{\mu\left(E_{i}\right)}$ in the measurable subsets of $E_{i} \times\{y\}$.

\section{Preliminaries and definitions}

In $\mathbb{R}^{n}$ we shall denote by $\mathbb{R}_{+}^{n}$ the positive orthant, and by $\left(\mathbb{R}_{+}^{n}\right)^{\circ}$ its interior. Also we shall denote by $\leq$ the usual order between numbers, and by $\ll$ the usual partial order between vectors in $\mathbb{R}^{n}$. Let $(X, \mathcal{A})$ be a measurable space.

Definition 2.1. (Murofushi and Sugeno [37]) A fuzzy measure on a measurable space $(X, \mathcal{A})$ is a set function $\mu: \mathcal{A} \rightarrow \mathbb{R}_{0}^{+}$with the properties:

- $\mu(\emptyset)=0 ; \mu(X)<+\infty ;$

- if $A \subset B$, then $\mu(A) \leq \mu(B)$ (monotonicity).

A fuzzy measure $\mu$ is subadditive if $\mu(A \cup B) \leq \mu(A)+\mu(B)$ for all elements $A, B$ from $\mathcal{A}$. A subadditive fuzzy measure will be also called a submeasure.

Submeasures are also called capacities, for an overview of the topic see for example [4, 40].

We now recall the concept of a semiconvex submeasure:

Definition 2.2. (See also [16, Lemma 2.1]) If $\mu: \mathcal{A} \rightarrow[0,1]$ is a fuzzy submeasure, we say that it is semiconvex if for every $A \in \mathcal{A}$ there exists a family of subsets of $A,\left(A_{t}\right)_{t \in[0,1]} \subset \mathcal{A}$ such that

2.2.i) $A_{0}=\varnothing, A_{1}=A$;

2.2.ii) $\mu\left(A_{t}\right)=t \mu(A)$;

2.2.iii) for $t<t^{\prime}$, there hold $A_{t} \subset A_{t^{\prime}}$ and $\mu\left(A_{t^{\prime}} \backslash A_{t}\right)=\left(t^{\prime}-t\right) \mu(A)$.

Definition 2.3. Given a fuzzy measure $\mu: \mathcal{A} \rightarrow \mathbb{R}_{0}^{+}$, (not necessarily sub-additive), we say that it is filtering if, for every element $A \in \mathcal{A}$ there exists an increasing family $\left(A_{t}\right)_{t \in[0,1]}$ of measurable subsets of $A$, such that (2.2,i)-(2.2, iiii) above hold true.

Remark 2.4. In this case, the range of $\mu_{A}$, namely of the measure $\mu$ restricted to $A$ is $[0, \mu(A)]$, for every measurable $A \subset X$. Moreover, given a family $\left\{\mu_{y}, y \in Y\right\}$ of fuzzy measures on $\mathcal{A}$, we say that they are uniformly filtering if for each element $A \in \mathcal{A}$ the same filtering family can be found, for all $\mu_{y}$.

A nontrivial example can be given as follows: Let $X_{0}=[0,1]$, and assume that $\mathcal{A}$ is the family of all subsets of $X_{0}$. It is well-known that there exist additive positive measures on $X_{0}$ (not $\sigma$-additive) extending the Lebesgue measure $\lambda$ to $\mathcal{A}$ : see e.g. [16, Theorem 1.3] and related bibliography [27, 38]; similar questions were discussed also in [21]. Denote by $\mu_{0}$ any of these measures, and set, for every $y \in] 0,1], \mu_{y}(A)=\mu_{0}(A)^{y}$ : clearly, $\mu_{y}$ is a fuzzy measure on $X_{0}$, not additive in general and it is filtering, because of continuity. Now, let $\left.\left.X=X_{0} \times\right] 0,1\right]$ and define, for each $E \subset X$

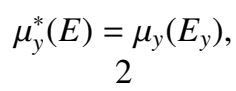


where $\left.E_{y}:=\left\{x \in X_{0}:(x, y) \in E\right)\right\}$. Clearly, for each $t \in[0,1]$, each $y$ and every $E$ there exists a subset $E_{t, y}^{\prime} \subset E_{y}$ such that $\mu_{y}\left(E_{t, y}^{\prime}\right)=t \mu_{y}\left(E_{y}\right)=t \mu_{y}^{*}(E)$. Thus, setting $E_{t}^{*}=\bigcup_{y \in] 0,1]} E_{t y}^{\prime}$ we get

$$
\mu_{y}^{*}\left(E_{t}^{*}\right)=\mu_{y}\left(E_{t, y}^{\prime}\right)=t \mu_{y}^{*}(E)
$$

as requested for uniform filtering.

We might be wondering if any classical $\sigma$-additive measure $\mu$ with range $[0, \mu(X)]$ has a filtering family satisfying (2.2 iiii). The answer is negative, since this condition for scalar measure is strictly related to the notion of continuity ([17, Definition 1.2]: for every $\varepsilon>0$ there exists a finite partition of $X: E_{1}, E_{2}, \ldots E_{k}$ such that $\mu\left(E_{i}\right) \leq \varepsilon$ for every $\left.i \leq k\right)$ and it does not follow in general from the additivity of the measure. In fact, if we consider $X=\mathbb{N}$ and the measure $\mu: \mathcal{P}(\mathbb{N}) \rightarrow[0,1]$ defined by $\mu(E)=\sum_{n=1}^{\infty} \frac{1_{E}(n)}{2^{n}}$; this measure is $\sigma$-additive with arcwiseconnected range $(R(\mu)=[0,1])$ but it is not continuous in the sense of [17, Definition 1.2] and so the condition (2.2)iiii) is not fulfilled. If the target space is infinite dimensional the situation is even worse, in fact the continuity does not imply the semiconvexity and then the convexity of the range ([17]).

\section{The Choquet integral and its properties}

Let $(X, \mathcal{A}, \mu)$ be a fuzzy measure space.

Definition 3.1. A function $f: X \rightarrow \mathbb{R}_{0}^{+}$is said to be measurable if the set $\{x \in X \mid f(x)>t\}$ is in $\mathcal{A}$ for every $t>0$. Any set of that type will be often denoted as $[f>t]$. The Choquet integral of a measurable function $f$ is defined by

$$
\int f d \mu:=\int_{0}^{\infty} \mu([f>t]) d t
$$

where the latter integral is in the Riemann sense. We say that $f \in L_{C}^{1}(\mu)$ if and only if $f$ is measurable and $\int f d \mu<\infty$.

This integral fulfills the following properties ([25, Proposition 5.1 and Chapter 11])

(3.1,i) $\int 1_{A} d \mu=\mu(A)$;

(3.1,ii) $\int c f d \mu=c \int f d \mu$ for $c \geq 0$;

(3.1,iii) if $f \leq g$ then $\int f d \mu \leq \int g d \mu$;

(3.1iv) $\int(f+c) d \mu=\int f d \mu+c \mu(X)$ for every $c \in \mathbb{R}^{+}$.

(3.1.v) if $\mu$ is subadditive then

$$
\int(f+g) d \mu \leq \int f d \mu+\int g d \mu ; \quad([25, \text { Theorem 6.3]) }
$$

3.1,vi) if $f, g$ are comonotonic, namely there is no pair $x, y \in X$ such that $f(x)>f(y)$ and $g(x)<g(y)$, then

$$
\int(f+g) d \mu=\int f d \mu+\int_{3} g d \mu ; \quad \text { (comonotonic additivity) }
$$


3.1,vii) for every $c>0$ it is

$$
\int f d \mu=\int \min \{f, c\} d \mu+\int(f-\min \{f, c\}) d \mu ;
$$

3.1.viii) for every non negative and $\mathcal{A}$-measurable function $f$ it is

$$
\int_{A} f d \mu:=\int f d \mu_{A}=\int f 1_{A} d \mu
$$

(see [25, chapter 11]); for arbitrary $f$ this last equality fails, as showed in [25, Example $11.1]$.

There is a huge literature concerning (3.1 vi) and its consequences; an interesting result on additivity is contained in [35] where the Sipoš and the Choquet integrals are compared and the additivity of the integrals are examined on some subspaces.

Now, we shall introduce a class of fuzzy measures, that in some sense can be considered as averages of other fuzzy measures on the space $X$. In particular, we shall assume the following, which will be kept for all the sequel.

Definition 3.2. Let $X^{*}:=X \times[0,1]$, and in $X^{*}$ let $\mathcal{H}$ be the $\sigma$-algebra $\mathcal{A} \times \mathcal{B}$, i.e. the product $\sigma$-algebra obtained by $\mathcal{A}$ and the Borel $\sigma$-algebra $\mathcal{B}$ in $[0,1]$. We say that a fuzzy measure $m: \mathcal{H} \rightarrow \mathbb{R}_{0}^{+}$is decomposable if, for every real number $y \in[0,1]$ there exists a non-trivial fuzzy measure $\mu_{y}: \mathcal{A} \rightarrow \mathbb{R}_{0}^{+}$, in such a way that the measures $\mu_{y}$ turn out to be equibounded and that

$$
m(H)=\int_{0}^{1} \mu_{y}\left(H_{y}\right) d y
$$

holds true, for all $H \in \mathcal{H}$. (Here the set $H_{y}$ is the $y$-section of $H$, and we implicitly assume that the mapping $y \mapsto \mu_{y}\left(H_{y}\right)$ is a measurable map, for all $\left.H\right)$.

From now on the measure $m$ will be decomposable.

Definition 3.3. Let $X^{*}:=X \times[0,1]$ be as above, and $f: X^{*} \rightarrow \mathbb{R}_{0}^{+}$be any mapping. We say that $f$ is sectional if there exists a measurable mapping $\varphi:[0,1] \rightarrow \mathbb{R}_{0}^{+}$such that

$$
f(x, y)=\varphi(y),
$$

for all $x$ and all $y$. If this is the case, we say that $\varphi$ is the section function of $f$. Usually, when this is the case, we shall also write $f(y)$ rather than $f(x, y)$, thus identifying $f$ and $\varphi$.

A kind of Fubini Theorem can be deduced for an arbitrary non-negative integrable mapping $f$, asserting that the integral of $f$ is obtained as an iterated one. We first prove a technical result of joint measurability for real-valued functions.

Lemma 3.4. Let us assume that $g:[a, b] \times[c, d] \rightarrow \mathbb{R}$ is any mapping, satisfying the following conditions:

(3.4)i) $g(t, \cdot)$ is measurable for all $t \in[a, b]$; 
(3.4 $[i i)$ there exists a finite measurable partition $\left\{E_{j}, j=1 \ldots k\right\}$ of the interval $[a, b]$ such that $g(t, y)=g\left(t^{\prime}, y\right)$ for all $t, t^{\prime} \in E_{j}$ and all $y$.

Then $g$ is jointly measurable in $(t, y)$.

Proof. For each index $j$ from 1 to $k$ choose arbitrarily a point $t_{j} \in E_{j}$. For each positive $\tau$, set

$$
A(\tau):=\{(t, y): g(t, y)>\tau\}:
$$

for each fixed $y$, the $y$-section $A(\tau)_{y}$ is an element of the finite algebra in $[a, b]$ generated by the sets $E_{j}$.

Now, denoting by $F_{1}, \ldots F_{K}$ the elements of this algebra, and setting $Y_{h}:=\left\{y: A(\tau)_{y}=F_{h}\right\}$, $h=1, \ldots, K$, we can see that $Y_{h}$ is measurable since it is a finite intersections of sets of the type $\left\{y \in[c, d]: g\left(t_{i}, y\right)>\tau\right\}$ for suitable values of $i$ and of the opposite type $\left\{y \in[c, d]: g\left(t_{i}, y\right) \leq \tau\right\}$ for the other indexes $i$, and in turn these sets are measurable since the mapping $g$ is separately measurable for each fixed $t$.

Finally, the formula

$$
A(\tau)=\bigcup_{h=1}^{k} F_{h} \times Y_{h}
$$

shows measurability of the set $A(\tau)$.

Theorem 3.5. Let $f: X^{*} \rightarrow \mathbb{R}_{0}^{+}$be any integrable map. Then we have

$$
\int_{X^{*}} f d m=\int_{0}^{1}\left(\int_{X} f(x, y) d \mu_{y}(x)\right) d y .
$$

Proof. We first assume that $f$ is bounded: $f(x, y) \leq M$ for all $(x, y) \in X^{*}$. For $(t, y) \in[0, M] \times$ $[0,1]$, consider the mapping

$$
g(t, y):=\mu_{y}(\{x: f(x, y)>t\}) .
$$

Of course, $g$ is decreasing in $t$. By means of dyadic partitions of $[0, M]$, (say $\left\{\left[t_{i}^{n}, t_{i+1}^{n}[, i=\right.\right.$ $\left.0, \ldots, 2^{n}-1\right\}$, where $\left.t_{i}^{n}=\frac{i}{2^{n}} M\right)$, it is easy to construct two sequences, $\left(g_{n}\right)_{n}$ and $\left(g^{n}\right)_{n}$ of step functions, such that for each $n$ and $y$ the mapping $g_{n}(t ; y)$ is constant in each dyadic interval, and equal to the value of $g(\cdot, y)$ at the right endpoint of the interval, while $g^{n}(t ; y)$ equals the value of $g(\cdot, y)$ at the left endpoint. In such a way, we have

$$
g_{n}(t ; y) \leq g_{n+1}(t ; y) \leq g(t, y) \leq g^{n+1}(t ; y) \leq g^{n}(t ; y)
$$

for all $n, y$ and $t$. We also point out that the mappings $g_{n}$ and $g^{n}$ (considered as depending on $t$ and $y$ ), are $\mathcal{B}^{2}$-measurable: this follows from Lemma 3.4 since [3.4-ii) is satisfied by construction an (3.4-i) follows from integrability of $f$ and definition of $m$.

Since $g$ is continuous in $t$ for all $t$ except a countable set, we can deduce that both sequences $\left(g_{n}\right)_{n}$ and $\left(g^{n}\right)_{n}$ converge to $g$ except for a countable set of values $t$ (possibly depending on $y$ ). Then, denoting by $\underline{g}$ and $\bar{g}$ respectively the limits of $g_{n}$ and $g^{n}$, and using dominated convergence, we see that

$$
\int_{0}^{M} \underline{g}(t ; y) d t=\int_{0}^{M} g(t ; y) d t=\int_{0}^{M} \bar{g}(t ; y) d t
$$


holds, for all $y$. Moreover, the functions $g$ and $\bar{g}$ are $\mathcal{B}^{2}$-measurable, as limits of sequences of mappings of this type. Thanks to Fubini's Theorem and to convergence in $L^{1}$, we now deduce that

$$
\begin{aligned}
\int_{X^{*}} f(x, y) d m & =\int_{0}^{M}\left(\int_{0}^{1} g(t ; y) d y\right) d t \geq \int_{0}^{M}\left(\int_{0}^{1} \underline{g}(t ; y) d y\right) d t= \\
& =\int_{0}^{1}\left(\int_{0}^{M} \underline{g}(t ; y) d t\right) d y=\int_{0}^{1}\left(\int_{0}^{M} g(t ; y) d t\right) d y= \\
& =\int_{0}^{1}\left(\int_{X} f(x, y) d \mu_{y}(x)\right) d y .
\end{aligned}
$$

On the other hand,

$$
\begin{aligned}
\int_{X^{*}} f(x, y) d m & =\int_{0}^{M}\left(\int_{0}^{1} g(t ; y) d y\right) d t \leq \int_{0}^{M}\left(\int_{0}^{1} \bar{g}(t ; y) d y\right) d t= \\
& =\int_{0}^{1}\left(\int_{0}^{M} \bar{g}(t ; y) d t\right) d y=\int_{0}^{1}\left(\int_{0}^{M} g(t ; y) d t\right) d y= \\
& =\int_{0}^{1}\left(\int_{X} f(x, y) d \mu_{y}(x)\right) d y .
\end{aligned}
$$

Comparing the two inequalities found, we obtain the assertion, for bounded $f$.

Now, if $f$ is unbounded, it is easy to reach the conclusion, by setting $f_{n}=f \wedge n$ for each integer $n$, and observing that

$$
\int_{X^{*}} f d m=\lim _{n} \int_{X^{*}} f_{n} d m
$$

and also

$$
\int_{X} f(x, y) d \mu_{y}(x)=\lim _{n} \int_{X} f_{n}(x, y) d \mu_{y}(x)
$$

for each fixed $y$, from which

$$
\int_{0}^{1}\left(\int_{X} f(x, y) d \mu_{y}(x)\right) d y=\lim _{n} \int_{0}^{1}\left(\int_{X} f_{n}(x, y) d \mu_{y}(x)\right) d y,
$$

by monotone convergence.

Corollary 3.6. Assume that $f: X^{*} \rightarrow \mathbb{R}_{0}^{+}$is sectional and integrable. Then we have, for each $H \in \mathcal{H}$ :

$$
\int_{H} f d m=\int_{0}^{1} f(y) \mu_{y}\left(H_{y}\right) d y
$$

where as usual $H_{y}$ denotes the $y$-section of $H$.

Proof. Let $f: X^{*} \rightarrow \mathbb{R}_{0}^{+}$be any sectional integrable map, $f(x, y)=f(y)$, and choose arbitrarily any measurable set $H \subset X^{*}$. Then

$$
\int_{H} f d m=\int_{X^{*}} f(y) 1_{H}(x, y) d m .
$$


Thanks to Theorem 3.5 , we get

$$
\int_{H} f d m=\int_{0}^{1}\left(\int_{H_{y}} f(y) d \mu_{y}(x)\right) d y,
$$

where $H_{y}=\{x \in X:(x, y) \in H\}$. Since the inner integrand is independent on $x$, we obtain

$$
\int_{H} f d m=\int_{0}^{1} \mu_{y}\left(H_{y}\right) f(y) d y,
$$

as announced.

Remark 3.7. Another consequence of Theorem 3.5 is the following.

If two integrable functions $f_{1}, f_{2}$ are comonotonic with respect to $x$ for every $y \in[0,1]$, then

$$
\begin{aligned}
\int_{X^{*}}\left(f_{1}+f_{2}\right) d m & =\int_{0}^{1}\left(\int_{X}\left[f_{1}(x, y)+f_{2}(x, y)\right] d \mu_{y}(x)\right)= \\
& =\int_{X^{*}} f_{1} d m+\int_{X^{*}} f_{2} d m .
\end{aligned}
$$

In particular, If $f_{1}, f_{2}$ are sectional and integrable functions and $f_{1} \pm f_{2} \geq 0$ then, for every $H \in \mathcal{H}$

$$
\int_{H}\left(f_{1} \pm f_{2}\right) d m=\int_{H} f_{1} d m \pm \int_{H} f_{2} d m
$$

For measurable vector functions $f: X^{*} \rightarrow \mathbb{R}_{+}^{n}$, the Choquet integral is defined componentwise, so it is an $n$-dimensional vector too. Assuming that $m$ is decomposable, we first prove the following result:

Proposition 3.8. For every constant vector $p \in \mathbb{R}_{+}^{n}$ and every integrable sectional function $f: X^{*} \rightarrow \mathbb{R}_{+}^{n}$, we have

$$
\int_{X^{*}} p \cdot f d m=p \cdot \int_{X^{*}} f d m .
$$

Proof. If $f$ is sectional then its components are sectional too and so it is enough to apply (3.2) of Remark 3.7

The additivity obtained in Propostion 3.8 can be extended to functions $f(x, y)=g(x) h(y)$ for suitable $g$ and $h$.

Proposition 3.9. For every constant vector $p \in \mathbb{R}_{+}^{n}$, every bounded measurable function $g$ : $X \rightarrow \mathbb{R}_{0}^{+}$, and every integrable vector function $h:[0,1] \rightarrow \mathbb{R}_{+}^{n}$, we have

$$
\int_{X^{*}} g(x) p \cdot h(y) d m=p \cdot \int_{X^{*}} g(x) h(y) d m .
$$

Proof. First, we observe that the conclusion can be easily obtained, when $g=c 1_{H}$, where $c$ is any positive real constant and $H$ is any measurable subset of $X$, i.e.

$$
\int_{X \times[0,1]} c 1_{H}(x) p \cdot h(y) d m=p \cdot \int_{X \times[0,1]} c 1_{H}(x) h(y) d m .
$$


Now, when $g$ is any simple function, with decreasing representation $g=\sum_{i} c_{i} 1_{H_{i}}$, one has

$$
\int_{X^{*}} g(x) p \cdot h(y) d m=\int_{0}^{1}\left(\int_{X} g(x) d \mu_{y}\right) p \cdot h(y) d y=\int_{0}^{1}\left(\sum_{i} c_{i} \mu_{y}\left(H_{i}\right)\right) p \cdot h(y) d y
$$

thanks to Theorem 3.5 But we have

$$
\begin{aligned}
\int_{0}^{1}\left(\sum_{i} c_{i} \mu_{y}\left(H_{i}\right)\right) p \cdot h(y) d y & =\sum_{i} \int_{0}^{1}\left(c_{i} 1_{H_{i}}(x) d \mu_{y}(x)\right) p \cdot h(y) d y= \\
& =p \cdot \int_{X^{*}} g(x) h(y) d m,
\end{aligned}
$$

by virtue of (3.3). Finally, if $g$ is any bounded measurable function, it can be uniformly approximated by an increasing sequence of simple functions $\left(g_{n}\right)_{n}$; then, by the properties of the Choquet integral, one has that

$$
\lim _{n} \int_{X^{*}} g_{n}(x) p \cdot h(y) d m=\int_{X^{*}} g(x) p \cdot h(y) d m,
$$

and finally

$$
\int_{X^{*}} g(x) p \cdot h(y) d m=p \cdot \int_{X^{*}} g(x) h(y) d m
$$

follows from the previous step.

Our next goal is to prove that, in case $f: X^{*} \rightarrow \mathbb{R}_{+}^{n}$ is sectional, and if $m$ is a decomposable fuzzy measure of a special type, then the set $R(f):=\left\{\int_{H} f d m: H \in \mathcal{H}\right\}$ is convex. We need the following

Definition 3.10. Let $m: \mathcal{H} \rightarrow \mathbb{R}_{0}^{+}$be a decomposable fuzzy measure. We shall say that $m$ is of convex type if the measures $\mu_{y}$ are uniformly filtering in the $\sigma$-algebra $\mathcal{A}$.

We observe that $m$ is of convex type if all measures $\mu_{y}$ coincide with a semiconvex submeasure $\mu$ on $X$, or in the particular case of a finite number of sections as described in the Introduction.

We shall also make use of the following Lemma.

Lemma 3.11. In the situation described above, let $\tau:[0,1] \rightarrow[0,1]$ be any measurable mapping. Then, there exists a measurable set $A \in \mathcal{H}$ such that

$$
\mu_{y}\left(A_{y}\right)=\tau(y) \mu_{y}(X)
$$

for all $y \in[0,1]$.

Proof. Since $0 \leq \tau \leq 1$, it is possible to construct an increasing sequence $\left(s_{k}\right)_{k}$ of simple functions converging to $\tau$, and a decreasing sequence $\left(S_{k}\right)_{k}$ of simple functions, also converging to $\tau$. Moreover, these sequences can be based on the same partitions of $[0,1]$, built in a diadic way. So we can write

$$
s_{k}=\sum_{i=0}^{2^{k}-1} c_{i}^{k} 1_{J_{i}^{k}}, \quad S_{k}=\sum_{i=0}^{2^{k}-1} C_{i}^{k} 1_{J_{i}^{k}},
$$


where $c_{i}^{k}=\frac{i}{2^{k}}, C_{i}^{k}=\frac{i+1}{2^{k}}$, and $J_{i}^{k}=\tau^{-1}\left(\left[\frac{i}{2^{k}}, \frac{i+1}{2^{k}}[)\right.\right.$ for all $k$ and $i=0 \ldots 2^{k}-1$.

Thanks to the convex-type hypothesis, there exists a filtering family $\left(X_{t}\right)_{t \in[0,1]}$ in $X$, satisfying (2.2-i,ii,iii) simultaneously for all the measures $\mu_{y}$. So we can set, for each $k$ :

$$
E_{k}:=\bigcup_{i=0}^{2^{k}-1} X_{c_{i}^{k}} \times J_{i}^{k}, \quad F_{k}:=\bigcup_{i=0}^{2^{k}-1} X_{C_{i}^{k}} \times J_{i}^{k}
$$

Clearly, the sets $E_{k}$ and $F_{k}$ belong to $\mathcal{H}$, and we have $E_{k} \subset E_{k+1} \subset F_{k+1} \subset F_{k}$, for all $k$. Moreover, we can see that

$$
\mu_{y}\left(E_{k, y}\right)=s_{k}(y) \mu_{y}(X), \mu_{y}\left(F_{k, y}\right)=S_{k}(y) \mu_{y}(X)
$$

for all $k$ and $y$.

Now, setting $E=\bigcup E_{k}, F=\bigcap F_{k}$, both $E$ and $F$ belong to $\mathcal{H}$, and $E \subset F$. By monotonicity, we have then

$$
\mu_{y}\left(E_{y}\right) \geq \sup _{k} \mu_{y}\left(E_{k, y}\right)=\lim _{k} s_{k}(y) \mu_{y}(X)=\tau(y) \mu_{y}(X),
$$

and

$$
\mu_{y}\left(F_{y}\right) \leq \inf _{k} \mu_{y}\left(F_{k, y}\right)=\lim _{k} S_{k}(y) \mu_{y}(X)=\tau(y) \mu_{y}(X) .
$$

Comparing these inequalities, and recalling that $E \subset F$, we can conclude that

$$
\mu_{y}\left(E_{y}\right)=\mu_{y}\left(F_{y}\right)=\tau(y) \mu_{y}(X)
$$

for all $y$. So, any of the sets $E$ or $F$ is as requested.

Theorem 3.12. Let's assume that $m$ is a fuzzy measure of convex type, and that $f: X^{*} \rightarrow \mathbb{R}_{+}^{n}$ is a sectional integrable function. Then the set $R(f)$ is convex.

Proof. As usual we shall identify $f$ with its (vector) section function, and fix any element $H \in \mathcal{H}$. We have, from Corollary 3.6:

$$
\int_{H} f d m=\int_{0}^{1} f(y) \mu_{y}\left(H_{y}\right) d y .
$$

More precisely, for any $y \in[0,1]$, set

$$
\Lambda(y)=\left(f_{1}(y) \mu_{y}\left(H_{y}\right), \ldots, f_{n}(y) \mu_{y}\left(H_{y}\right)\right):
$$

clearly, $\Lambda$ is measurable, and $\int_{H} f d m=\int_{0}^{1} \Lambda(y) d y$. Since $\mu_{y}\left(H_{y}\right) \leq \mu_{y}(X)$, the vector $\Lambda(y)$ lies in the line segment joining the origin $O$ with the vector $\mu_{y}(X) f(y)$. So, if we denote this segment by $S(y)$, it is clear that $\int_{H} f d m \in \int_{0}^{1} S(y) d y$, where the latter is an Aumann integral, i.e. the set of all integrals of measurable selections from $y \mapsto S(y)$ : from now on, we shall denote by $D$ this Aumann integral. Since $D$ is clearly convex, if we prove that $R(f)=D$ the proof is complete. But we have already seen that $R(f) \subset D$, so it only remains to show the converse. To this aim, let us fix any measurable selection $\sigma(y)$ from $S(y)$. Then, for all $y$ there exists a real number 
$\tau(y) \in[0,1]$ such that (componentwise)

$$
\sigma_{i}(y)=\tau(y) \mu_{y}(X) f_{i}(y) .
$$

The mapping $\tau$ can be taken measurable: indeed, let $i$ be any index for which $f_{i}(y) \neq 0$; then $\tau(y)=\frac{\sigma_{i}(y)}{\mu_{y}(X) f_{i}(y)}$ and this value is independent of $i$. Otherwise, if $f_{i}(y)=0$ we can choose $\tau$ as in the previous case, since its value is immaterial.

Now, since $0 \leq \tau \leq 1$, thanks to Lemma 3.11, we can find a measurable set $E \in \mathcal{H}$ such that $\mu_{y}\left(E_{y}\right)=\tau(y) \mu_{y}(X)$ for all $y \in[0,1]$, and so, componentwise:

$$
\int_{E} f_{i} d m=\int_{0}^{1} f_{i}(y) \mu_{y}\left(E_{y}\right) d y=\int_{0}^{1} f_{i}(y) \tau(y) \mu_{y}(X) d x=\int_{0}^{1} \sigma_{i}(y) d y .
$$

Since $\sigma$ was arbitrary, this shows that $D \subset R(f)$, and the proof is finished.

\section{Applications to equilibria}

We shall now introduce our economic model. For vector measurable functions $f=\left(f_{1}, \ldots f_{n}\right)$ : $X^{*} \rightarrow \mathbb{R}_{+}^{n}$ we consider the monotone integral, and we shall keep the notation $\int f d m$, as the vector $\int f d m=\left(\int f_{1} d m, \ldots, \int f_{n} d m\right)$. Sometimes we shall denote by $a$ the generic element $(x, y) \in X^{*}$, and shall also use the notation $f \in L_{C}^{1}\left(m, \mathbb{R}_{+}^{n}\right)$ meaning that each component is in $L_{C}^{1}(m)$.

We define a pure exchange economy to be a 4-tuple

$$
\mathcal{E}=\left\{\left(X^{*}, \mathcal{H}, m\right) ; \mathbb{R}_{+}^{n} ; e ;\left\{>_{a}\right\}_{a \in X^{*}}\right\},
$$

where:

- the space of agents is a triple $\left(X^{*}, \mathcal{H}, m\right)$, with $\left(X^{*}, \mathcal{H}\right)$ a measurable space and $m$ is a fuzzy measure of convex type. Moreover we shall require that each $m$ is a submodular and the ideal of $m$-null sets is stable under countable unions. Under these conditions (see [24, 41]) the Choquet integral for scalar non-negative functions satisfies the following requirements:

(c) $\int_{X^{*}}(f+g) d m \leq \int_{X^{*}} f d m+\int_{X^{*}} g d m$

(c) If $\int_{A} f d m \leq \int_{A} g d m$ for every $A \in \mathcal{H}$ then $f \leq g m$-a.e.

We shall also assume here that $\mu_{y}(X)=m\left(X^{*}\right)=1$ for all $y \in[0,1]$.

-the target space $\mathbb{R}^{n}$ is the commodity space, and its positive cone $\mathbb{R}_{+}^{n}$ is called the consumption set of each agent;

- $e: X^{*} \rightarrow \mathbb{R}_{+}^{n}, e \in L_{C}^{1}\left(m, \mathbb{R}_{+}^{n}\right)$ is the initial endowment density and $e(a):=e(x, y) \gg 0 m$-a.e.; we shall always assume that $e$ is sectional.

$-\left\{>_{a}\right\}_{a}$ is the preference relation associated to the generic agent $a \in X^{*}$.

Let us introduce the classical concepts of equilibrium theory in this new setting.

- An allocation is a measurable function $f: X^{*} \longrightarrow \mathbb{R}_{+}^{n}$; an allocation is feasible if

$$
\int_{X^{*}} f d m=\int_{10} e d m .
$$


- A price is any element $p \in \mathbb{R}_{+}^{n} \backslash\{0\}$.

- The budget set of an agent $a \in X^{*}$ for the price $p$ is $B_{p}(a)=\left\{x \in \mathbb{R}_{+}^{n}: p x \leq p e(a)\right\}$.

- A coalition is any measurable subset $S$ of $X^{*}$ with $m(S)>0$.

- We say that the coalition $S$ can improve the allocation $f$ if there exists an allocation $g$ such that

(i $\left.i_{1}\right) g(a)>_{a} f(a) m$-a.e. in $S$;

(i $\left.i_{2}\right) \int_{S} g d m=\int_{S} e d m$.

- The core of $\mathcal{E}$, denoted by $C(\mathcal{E})$, is the set of all the feasible allocations that cannot be improved by any coalition.

- We say that the coalition $S$ strongly improves the allocation $f$ if there exists an allocation $g$ such that

(i $\left.i_{1}\right) g(a)>_{a} f(a) m$-a.e. in $S$;

$\left(i_{2}^{\prime}\right) \int_{S_{y}} g(\cdot, y) d \mu_{y}=\int_{S_{y}} e(\cdot, y) d \mu_{y}$ for a.e. $y \in[0,1]$.

- The large core of $\mathcal{E}$, denoted by $L C(\mathcal{E})$, is the set of all the feasible allocations that cannot be strongly improved by any coalition. Of course, $C(\mathcal{E}) \subset L C(\mathcal{E})$.

- A Walras equilibrium of $\mathcal{E}$ is a pair $(f, p) \in L_{C}^{1}\left(m, \mathbb{R}_{+}^{n}\right) \times\left(\mathbb{R}_{+}^{n} \backslash\{0\}\right)$ such that:

$\left(w_{1}\right) f$ is a feasible allocation;

( $\left.w_{2}\right) f(a)$ is a maximal element of $>_{a}$ in the budget set $B_{p}(a)$, (namely $f(a) \in B_{p}(a)$ and $x>_{a}$ $f(a)$ implies $p \cdot x>p \cdot e(a))$ for $m$-almost all $a \in X^{*}$.

- A walrasian allocation is a feasible allocation $f$ such that there exists a price $p$ so that the pair $(f, p)$ is a Walras equilibrium. $W(\mathcal{E})$ is the set of all the walrasian allocations of $\mathcal{E}$.

Our aim is to obtain relations between Walras equilibria $\mathcal{W}(\mathcal{E})$ and core of an economy $\mathcal{C}(\mathcal{E})$.

Assumption 4.1. (4.1.1) (Perfect competition) $m$ is a fuzzy measure of convex type, and the corresponding measures $\mu_{y}$ are sub-additive. This condition describes an economy where the big coalition is the average of many autonomous sections.

(4.1,2) $e: X^{*} \rightarrow\left(\mathbb{R}_{+}^{n}\right)^{\circ}$ is sectional, and $\varphi^{e}$ will denote its (vector) section function. Observe that this implies that the aggregate initial endowment $\int_{X^{*}} e(a) d m \in\left(\mathbb{R}_{+}^{n}\right)^{\circ}$. Let $\lambda: \mathcal{H} \rightarrow \mathbb{R}_{+}^{n}$ defined by $\lambda(H)=\int_{H}$ edm. Then $\lambda$ is a fuzzy subadditive measure.

(4.1,3) In $\mathbb{R}_{+}^{n}$ there exist preorders $>_{a}, a \in X^{*} \times[0,1]$, that satisfy the following:

(4.133a) (Monotonicity) for every $x \in \mathbb{R}_{+}^{n}$ and every $v \in \mathbb{R}_{+}^{n} \backslash\{0\}, x+v>_{a}$ x for all $a \in X^{*}$.

[4.13b) continuity, namely for all $x \in\left(\mathbb{R}_{+}^{n}\right)^{\circ}$ the set $\left\{y \in \mathbb{R}_{+}^{n}: y \geq_{a} x\right\}$ is closed in $\mathbb{R}_{+}^{n}$ for all $a \in X^{*}$;

and such that for every $y$ and $a \in X \times y, \geq_{a}$ is the same preorder depending only on $y$, i.e. $x \geq_{a} x^{\prime}$ can be written as $x \geq_{y} x^{\prime}$. In other words, in each coalition $E_{y}:=X \times\{y\}$, agents share the same initial endowment and the same preference criterion. 
In order to study relations between $C(\mathcal{E})$ and $\mathcal{W}(\mathcal{E})$, we observe that

Theorem 4.2. Under the assumptions 4.1 the inclusion $C(\mathcal{E}) \supset W(\mathcal{E})$ holds true.

Proof. The proof is analogous to [41, Theorem 3.2] and it is reported here for the sake of completeness. Let $f \in W(\mathcal{E}) \backslash C(\mathcal{E})$. Then there exist a coalition $S$ and a feasible allocation $g$ such that $m$-a.e. in $S g(a)>_{a} f(a)$ and $\int_{S} g d m=\int_{S} e d m$. On the other side there exists a price $p$ for which $f(a)$ is $>_{a}$ maximal in $B_{p}(a) m$-a.e. in $S$.

Consequently, setting $S_{1}=\{a \in S: p \cdot g(a) \leq p \cdot e(a)\}$, it should be $m\left(S_{1}\right)=0$, otherwise the function $\widetilde{f}=g 1_{S_{1}}+f 1_{S \backslash S_{1}}$ would contradict the maximality of $f$.

Hence $m$-a.e. in $S$ one has:

$$
p \cdot g(a)=\sum_{i=1}^{n} p_{i} g_{i}(a)>\sum_{i=1}^{n} p_{i} e_{i}(a)=p \cdot e(a)
$$

whence, by Proposition 3.8

$$
\int_{S} p \cdot g(a) d m=\int_{S} \sum_{i=1}^{n} p_{i} g_{i}(a) d m>\int_{S} \sum_{i=1}^{n} p_{i} e_{i}(a) d m=p \cdot \int_{S} e d m .
$$

Thus

$$
\int p \cdot g d m>p \cdot \int e d m
$$

On the other side, as we have assumed that $g$ improves $f$, from subadditivity we have:

$$
\int_{S} p \cdot g d m \leq p \cdot \int_{S} g(a) d m=p \cdot \int_{S} e d m
$$

thus contradicting (4.1).

Assumption 4.3. Suppose now that $f$ is sectional, so that $f(a)=f(x, y)=f(y)$ and consider the multifunction

$$
\Gamma_{f}(a):=\Gamma_{f}(x, y)=\left\{t \in \mathbb{R}_{+}^{n}: t \geq_{a} f(a)\right\}=\left\{t \in \mathbb{R}_{+}^{n}: t \geq_{y} f(y)\right\}=C_{y},
$$

where the $C_{y}$ 's are convex, closed and contain the sets $u+\left(\mathbb{R}_{+}^{n}\right)^{\circ}$ when $u \in C_{y}$. The class of its Choquet integrable selections is $S_{\Gamma_{f}}^{*}=\left\{\psi \in L_{C}^{1}\left(m, \mathbb{R}_{+}^{n}\right)\right.$ with $\psi(a) \in \Gamma_{f}(a)$ for $m-$ a.e. $\left.a \in X\right\}$.

So $\Gamma_{f}$ contains as selections all functions that are $\mu_{y}$-a.e. constant in $X \times\{y\}$ (the constant must be an element of $\left.C_{y}\right)$. So all integrable functions of the type $\gamma(x, y)=c(y), c(y) \in C_{y}$, are Choquet integrable selections of $\Gamma$.

Let

$$
I_{f}:=\left\{z=\int_{H} s d m-\lambda(H), \quad \forall H \in \mathcal{H}, s \in S_{\Gamma_{f}}^{*}\right\}
$$


Now, in order to prove the convexity of $I_{f}$ we need some preliminary results; the first is a density result of the multivalued integral of $\Gamma$.

Lemma 4.4. If $s \in S_{\Gamma_{f}}^{*}$ then, for every $A \in \mathcal{H}$, there exists a sectional selection $g \in S_{\Gamma_{f}}^{*}$ such that

$$
\int_{A} s d m=\int_{A} g d m
$$

Proof. Let $A \in \mathcal{H}$ and $s \in S_{\Gamma}^{*}$ be fixed. For each $y$, let us define

$$
\varphi(y)=\frac{1}{\mu_{y}\left(A_{y}\right)} \int_{A_{y}} s(x, y) d \mu_{y}(x),
$$

in case $\mu_{y}\left(A_{y}\right)>0$. Otherwise we can set $\varphi(y)$ equal to any arbitrary selection of $y \mapsto C_{y}$. Indeed, we see that

$$
\begin{aligned}
\int_{A} s d m & =\int_{K}\left(\int_{A_{y}} s(x, y) d \mu_{y}(x)\right) d y+\int_{K^{c}}\left(\int_{A_{y}} s(x, y) d \mu_{y}(x)\right) d y= \\
& =\int_{K}\left(\int_{A_{y}} s(x, y) d \mu_{y}(x)\right) d y,
\end{aligned}
$$

where $K$ denotes the set of all $y \in[0,1]$ such that $\mu_{y}\left(A_{y}\right)>0$. Then, setting $g(x, y)=\varphi(y)$, we have

$$
\int_{A} g(x, y) d m=\int_{0}^{1} \varphi(y) \mu_{y}\left(A_{y}\right) d y
$$

thanks to Corollary 3.6 and so

$$
\int_{A} g(x, y) d m=\int_{K} \varphi(y) \mu_{y}\left(A_{y}\right) d y=\int_{K}\left(\int_{A_{y}} s(x, y) d \mu_{y}(x)\right) d y=\int_{A} s d m .
$$

It only remains to show that $\varphi(y) \in C_{y}$, for each $y \in K$. To this aim, fix $y \in K$ and assume by contradiction that the quantity $\varphi(y)$ does not belong to $C_{y}$. Then, by the Separation Theorem, there exist a positive element $p \in \mathbb{R}^{n}$ and a real number $a$ such that

$$
p \cdot \varphi(y)<a, \quad \inf \left\{p \cdot z: z \in C_{y}\right\} \geq a .
$$

But then, by subadditivity, we get

$$
a>p \cdot \varphi(y)=\sum_{i=1}^{n} p_{i} \frac{1}{\mu_{y}\left(A_{y}\right)} \int_{A_{y}} s_{i}(x, y) d \mu_{y}(x) \geq \frac{1}{\mu_{y}\left(A_{y}\right)} \int_{A_{y}} \sum_{i=1}^{n} p_{i} s_{i}(x, y) d \mu_{y}(x) .
$$

Now, for every $x \in X$ we have $s(x, y) \in C_{y}$, and so

$$
\sum_{i=1}^{n} p_{i} s_{i}(x, y) \geq a:
$$


from this we deduce

$$
a>p \cdot \varphi(y) \geq \frac{1}{\mu_{y}\left(A_{y}\right)} \int_{A_{y}} \sum_{i=1}^{n} p_{i} s_{i}(x, y) d \mu_{y}(x) \geq \frac{1}{\mu_{y}\left(A_{y}\right)} \int_{A_{y}} a d \mu_{y}(x)=a .
$$

This is clearly absurd, and the assertion is proved.

Using Lemma4.4 we now prove the main convexity theorem.

Theorem 4.5. The set $I_{f}$ is convex, when $f$ satisfies Assumption 4.3

Proof. Thanks to Lemma4.4 in the definition of $I_{f}$ the mapping $s$ can be taken sectional, without loss of generality. So we always assume this, and write the values of $s$ just as $s(y)$, subject to the condition $s(y) \in C_{y}$ for all $y$. Our aim is to prove that

$$
I_{f}=\widehat{\int}_{0}^{1} I(y) d y,
$$

where $I(y)$ is the convex cone $\left(C_{y}-e(y)\right) \cdot[0,1]$, and the multivalued integral is meant as follows:

$$
\widehat{\int}_{0}^{1} I(y) d y=\left\{\int_{0}^{1}(s(y)-e(y)) \tau(y) d y: s \in S_{\Gamma_{f}}^{*}, \tau(y) \in[0,1], s, \tau \text { integrable }\right\} .
$$

Indeed, if $z \in I$ there exist an integrable selection $s$ and a set $A \in \mathcal{H}$ such that

$$
z=\int_{A} s d m=\int_{0}^{1}(s(y)-e(y)) \mu_{y}\left(A_{y}\right) d y .
$$

By definition of $m$, the mapping $y \mapsto \mu_{y}\left(A_{y}\right)$ is measurable, and obviously bounded between 0 and $\mu_{y}(X)=1$, so clearly $z \in \widehat{\int}_{0}^{1} I(y) d y$.

Conversely, let us take an element $w \in \widehat{\int}_{0}^{1} I(y) d y$ : then there exist a measurable selection $s$ and a measurable mapping $\tau:[0,1] \rightarrow[0,1]$, such that

$$
w=\int_{0}^{1}(s(y)-e(y)) \tau(y) d y .
$$

Thanks to Lemma 3.11, we see that there exists a measurable set $A \in \mathcal{H}$ such that $\tau(y)=\mu_{y}\left(A_{y}\right)$ for all $y$, and so

$$
w=\int_{0}^{1}(s(y)-e(y)) \mu_{y}\left(A_{y}\right) d y=\int_{A}(s-e) d m \in I_{f} .
$$

This proves also the reverse inclusion. Now, we shall prove that $\widehat{\int}_{0}^{1} I(y) d y$ is convex. So, take two elements $w_{1}$ and $w_{2}$ from $\widehat{\int}_{0}^{1} I(y) d y$, and fix any positive number $\left.c \in\right] 0,1[$. By definition, there exist measurable selections $s_{i}$ and measurable mappings $\tau_{i}:[0,1] \rightarrow[0,1], i=1,2$, such 
that

$$
w_{i}=\int_{0}^{1}\left(s_{i}-e_{i}\right)(y) \tau_{i}(y) d y
$$

$i=1,2$. Define

$$
K:=\left\{y \in[0,1]: \tau_{1}(y)+\tau_{2}(y) \neq 0\right\} .
$$

Then we have

$$
\begin{aligned}
c w_{1}+(1-c) w_{2} & =\int_{K}\left[c\left(s_{1}-e\right)(y) \tau_{1}(y)+(1-c)\left(s_{2}-e\right)(y) \tau_{2}(y)\right] d y= \\
& =\int_{K}\left(c \tau_{1}(y)+(1-c) \tau_{2}(y)\right) \cdot\left[\frac{c \tau_{1}(y)\left(s_{1}(y)-e(y)\right)}{c \tau_{1}(y)+(1-c) \tau_{2}(y)}+\right. \\
& \left.+\frac{(1-c) \tau_{2}(y)\left(s_{2}(y)-e(y)\right)}{c \tau_{1}(y)+(1-c) \tau_{2}(y)}\right] d y
\end{aligned}
$$

Let us set

$$
\tau(y)= \begin{cases}c \tau_{1}(y)+(1-c) \tau_{2}(y), & y \in K \\ 0, & y \notin K\end{cases}
$$

and

$$
s(y)= \begin{cases}\frac{c \tau_{1}(y) s_{1}(y)}{c \tau_{1}(y)+(1-c) \tau_{2}(y)}+\frac{(1-c) \tau_{2}(y) s_{2}(y)}{c \tau_{1}(y)+(1-c) \tau_{2}(y)} & y \in K \\ s_{1}(y) & y \notin K .\end{cases}
$$

Then, it is clear that $\tau$ is a measurable mapping with values in $[0,1]$ and $s$ is a measurable function, such that $s(y) \in C_{y}$ for all $y$ : moreover, it is clear from the previous calculations that

$$
c w_{1}+(1-c) w_{2}=\int_{0}^{1}(s(y)-e(y)) \tau(y) d y \in \widehat{\int}_{0}^{1} I(y) d y .
$$

So, we have proved also that the set $\widehat{\int}_{0}^{1} I(y) d y$ is convex, which concludes the proof.

In analogy with our previous notation, let $R(\lambda)$ denote the range of the set function $\lambda$, namely $R(\lambda)=\lambda(\mathcal{A})$.

Lemma 4.6. If $f \in L C(\mathcal{E})$, there exists $p \in \mathbb{R}_{+}^{n}, p \neq 0$ such that $p \cdot x \geq 0$ for all $x \in \bar{I}_{f}$.

Proof. We shall first prove that $I_{f} \cap\left(-\mathbb{R}_{+}^{n}\right)=\{0\}$. Indeed, assume by contradiction that there exists $z \in I_{f} \cap\left(-\mathbb{R}_{+}^{n}\right)$ with $z \neq 0$. Then there exist a coalition $A \in \mathcal{H}$ and a Choquet integrable selection $s \in S_{\Gamma_{f}}^{1}$ such that

$$
z=\int_{A} s d m-\int_{A} e d m \in\left(-\mathbb{R}_{+}^{n}\right) .
$$

Thanks to Lemma 4.4 we can and do assume that $s$ is sectional. Then immediately we get $m(A)>$ 0 (otherwise both $\int_{A} s d m=0$ and $\int_{A} e d m=0$ whence $z=0$ ). We observe that $z=\int_{0}^{1} z_{y} d y$, where $z_{y}=\int_{A_{y}} s(x, y) d \mu_{y}-e(y) \mu_{y}\left(A_{y}\right)=(s(y)-e(y)) \mu_{y}\left(A_{y}\right)$. 
Now, let $J:=\left\{y: z_{y} \neq 0\right\}$. Of course, $\lambda(J) \neq 0$ otherwise $z=0$, and $z=\int_{J} z_{y} d y$. Now, for each $y \in J$, we have $\mu_{y}\left(A_{y}\right)>0$ (otherwise $z_{y}=0$ ), and let us define $A^{\prime}:=\bigcup_{y \in J} A_{y}$ : the set $A^{\prime}$ is a measurable subset of $X^{*}$, since $J$ and $A$ are. Finally let us set

$$
s_{0}(x, y):=s(y)-\frac{z_{y}}{\mu_{y}\left(A_{y}\right)},
$$

for all $y \in J$ and $x \in X$. Since $z_{y} \in\left(-\mathbb{R}_{+}^{n}\right) \backslash\{0\}$ for each $y$, we see that the allocation $s_{0}$ satisfies $s_{0}(a)>_{a} s(a) \geq_{a} f(a) \mu$-a.e. in $A^{\prime}$, and moreover

$$
\int_{A_{y}^{\prime}} s_{0} d \mu_{y}=\int_{A_{y}^{\prime}} s(y) d \mu_{y}-z_{y}=e^{y} \mu_{y}\left(A_{y}\right)
$$

holds true, for all $y \in J$. Moreover, if $y \notin J$, we have by definition $A_{y}^{\prime}=\emptyset$, and so $\int_{A_{y}^{\prime}} s_{0} d \mu=0=\int_{A_{y}^{\prime}} e d \mu$. So we have proved that the coalition $A^{\prime}$ strongly improves $f$ by the allocation $s_{0}$. But this is impossible, since $f \in L C(\mathcal{E})$.

In conclusion $I_{f} \cap\left(-\mathbb{R}_{+}^{n}\right)=\{0\}$ and hence $\bar{I}_{f} \cap\left(-\mathbb{R}_{+}^{n}\right)^{o}=\emptyset$. Since both sets are convex, and the second one has non-empty interior, we can apply the Strong Separation Theorem, and determine some $p \in \mathbb{R}^{n} p \neq 0$ such that $p \cdot z \geq 0$ for all $z \in \bar{I}_{f}$.

It only remains to prove that $p \in \mathbb{R}_{+}^{n}$. Indeed, we have that $\left(\mathbb{R}_{+}^{n}\right)^{o} \subset I_{f}$; in fact if $z \in\left(\mathbb{R}_{+}^{n}\right)^{o}$, then the allocation $\psi=\frac{z}{m\left(X^{*}\right)}+f$ is in $S_{\Gamma_{f}}^{*}$ and

$$
\int_{X^{*}} \psi d m-\int_{X^{*}} e d m=\int_{X^{*}} f d m+z-\int_{X^{*}} e d m=z
$$

since $f$ is feasible. Then $p \cdot z \geq 0$ for every $z \in\left(\mathbb{R}_{+}^{n}\right)^{o}$, whence necessarily $p \in \mathbb{R}_{+}^{n}$.

We shall now prove that:

Lemma 4.7. (Strassen) If $f$ is sectional and $f \in L C(\mathcal{E})$, then $p \cdot e(a) \leq p \cdot f(a) m$-a.e., where $p$ is as in Lemma 4.6

Proof. Let $B:=\{y \in[0,1]: p \cdot f(y)<p \cdot e(y)\}$. Since $\mu_{y}(X)=m\left(X^{*}\right)=1$ and $f$ and $e$ are sectional, we have

$$
0<\int_{B}(p \cdot e-p \cdot f)(y) d y=\int_{X \times B}(p \cdot e-p \cdot f) d m .
$$

So, by Corollary 3.6 and Remark 3.7 it follows

$$
0<\int_{X \times B} p \cdot(e-f) d m=p \cdot \int_{X \times B} e d m-p \cdot \int_{X \times B} f d m=-p \cdot z,
$$
where $z=\int_{X \times B} f d m-\int_{X \times B} e d m$ is an element of $I_{f}$. Therefore $p \cdot z<0$, which is in contrast with
Lemma 4.6.

Theorem 4.8. Under the previous assumptions, if $f$ is a sectional allocation, then $f \in L C(\mathcal{E})$ if and only if $f \in W(\mathcal{E})$. 
Proof. We have already seen that $W(\mathcal{E}) \subset C(\mathcal{E})$, thanks to Theorem 4.2. To prove the converse inclusion fix $f \in L C(\mathcal{E})$. By Lemma 4.7 $m$ a.e. in $X^{*}, p \cdot e(a) \leq p \cdot f(a)$. We shall now prove that the previous inequality is in fact an equality. For $A \subset X^{*}$, being $f$ feasible and since $p \cdot f-p \cdot e \geq 0$ $m$-a.e., we derive by Proposition 3.8 and (3.2)

$$
0 \leq \int_{A} p \cdot(f-e) d m \leq \int_{X^{*}} p \cdot(f-e) d m=p \cdot\left(\int_{X^{*}} f d \mu-\int_{X^{*}} e d m\right) .
$$

Applying $\left(c_{2}\right)$ (pag. 10), we get $p \cdot f=p \cdot e m$-a.e. in $X$.

The remaining part of the proof is exactly the same as that of [28, Theorem 2.1.1, pag $133 \mathrm{ff}]$ since preferences are assumed to be monotone and continuous.

Assume now that the preferences have the following structure:

- for every $y \in[0,1]$, there exists a subset $J_{y}$ of $\{1,2, \ldots, n\}$ such that:

i) for every $u, v \in \mathbb{R}_{+}^{n}, u>_{a=(x, y)} v \Longleftrightarrow u_{j}>v_{j}, j \in J_{y}$;

ii) For every $j \in\{1, \ldots, n\}$, the set $A_{j}:=\left\{y \in[0,1]: j \in J_{y}\right\}$ is measurable.

This means that within each coalition $E_{k}$ only the items of the $k$-th list $J_{k}$ are considered, in order to decide whether a bundle is preferred to another. Observe that such assumption does not fulfill monotonicity, in the sense of (4.13b), but it satisfies the more demanding form

- for every $x \in \mathbb{R}_{+}^{n}, z \in\left(\mathbb{R}_{+}^{n}\right)^{0}$, then $x+z>_{a} x$ for every $a \in X$.

However Lemma 4.6 remains true: one has only to note that $I \cap\left(-\mathbb{R}_{+}^{n}\right)^{0}=\emptyset$ with the same proof.

Proposition 4.9. Under Assumption 4.1] $e \in W(\mathcal{E})$.

Proof. The proof is analogous to that of [41, Proposition 3.13] when we apply Theorem 4.8. It is enough to prove that $e \in C(\mathcal{E})$ and then apply Theorem 4.8. Assume by contradiction that $e \notin C(\mathcal{E})$; then there exists a pair $(f, S)$ that improves $e$, namely

4.9. a) $f>_{a} e$, when $a \in S$;

4.9.b) $\int_{S}^{*} f d m=\int_{S}^{*} e d m$.

We note that, from (i) and (ii) above, it follows that there exists $k \in\{1, \ldots, n\}$ such that $\lambda\left(A_{k}\right)>0$. From $4.9 \mathrm{a}$ ), we have for the $k$-th entries of $f$ and $e, f_{k}(a)>e_{k}(a), a \in S$. Hence by $\left(c_{2}\right)$, there holds

$$
\int_{S}^{*} f_{k} d m>\int_{S}^{*} e_{k} d m
$$

that contradicts $4.9 \mathrm{~b}$ ).

\section{Conclusions}

The Choquet integral over a product space $X^{*}$ and with values in $\mathbb{R}_{+}^{n}$ has been studied with respect to a fuzzy measure. Under suitable conditions a Fubini theorem is obtained and these results are used to find equilibria in a pure exchange economy $\mathcal{E}=\left\{\left(X^{*}, \mathcal{H}, m\right) ; \mathbb{R}_{+}^{n} ; e ;\left\{>_{a}\right\}_{a \in X^{*}}\right\}$, 
where the space of agents is a triple $\left(X^{*}, \mathcal{H}, m\right)$, with $\left(X^{*}, \mathcal{H}\right)$ a measurable space and $m$ is a fuzzy measure of convex type.

If the target space is infinite dimensional vector lattices are candidates for the space of goods. In this framework one could consider also the methods of integration given in $[12,13,18,19,24$, 26, 42].

\section{Acknowledgement}

This research was partially supported by GNAMPA - INDAM (Italy) Grant N. U UFMBAZ2017/0000326, by University of Perugia - Dept. of Mathematics and Computer Sciences Grant Nr 2010.011.0403 and Grant APVV-14-0013.

\section{References}

[1] L. Angeloni - A. Martellotti A separation result with applications to Edgeworth equivalence in some infinite dimensional setting, Commentationes Math. 44 (2004) 227-243.

[2] L. Angeloni - A. Martellotti Core-Walras equivalence in finitely additive economies with extremely desirable commodities, Mediterr. J. Math. 4, (2007). 87-107.

[3] T.E. Armstrong - M.K. Richter, The Core-Walras equivalence, J. Econom. Theory, 33, (1984), 116-151.

[4] G. Barbieri - A. Boccuto, On extensions of $k$-subadditive lattice group valued capacities, Italian Journal of Pure and Applied Math. 37, (2017), 387-408.

[5] A. Basile, Finitely additive nonatomic coalition production economies: core-Walras equivalence, Internat. Econom. Rev. 34, (1993), 983-995.

[6] A. Basile - M.G. Graziano, On the Edgeworth's conjecture in finitely additive economies with restricted coalitions, J, Mathematical Economics, 36 (3), (2001), 219-240.

[7] A. Basile - C. Donnini - M.G. Graziano, Core and equilibria in coalitional asymmetric information economies, J. of Mathematical Economics, 45, (2009), 293-307.

[8] A. Basile - C. Donnini - M.G. Graziano, Core equivalences for equilibria supported by non-linear prices, Positivity 17 (3), (2013), 621-653.

[9] P. Benvenuti - R. Mesiar, A note on Sugeno and Choquet integrals in: Proceedings of the IPMU, Madrid, 2000, pp. 582-585.

[10] P. Benvenuti - R. Mesiar, Integrals with respect to a general fuzzy measure in: M. Grabisch et al. Fuzzy Measures and Integrals, Theory and applications Stud. Fuzziness Soft Computer, 40, (2000) Physica-Verlag, Heidelberg, pp. 205-232.

[11] P. Benvenuti - R. Mesiar - D. Vivona, Monotone set functions-based integrals, in: E. Pap (Ed.), Handbook of Measure Theory, vol. II, Elsevier, (2002), 1329-1379.

[12] A. Boccuto - D. Candeloro - A.R. Sambucini, Henstock multivalued integrability in Banach lattices with respect to pointwise non atomic measures, Atti Accad. Naz. Lincei Rend. Lincei Mat. Appl. 26, (2015), 363-383

[13] A. Boccuto - A.M. Minotti - A.R. Sambucini, Set-valued Kurzweil-Henstock integral in Riesz space setting, PanAmerican Mathematical Journal, 23 (1) (2013), 57-74.

[14] A. Boccuto - A.R. Sambucini, On the De Giorgi-Letta integral with respect to means with values in Riesz spaces, Real Analisys Exchange 21 (2), (1995/96), 793-810.

[15] A. Boccuto - A.R. Sambucini, Comparison between different types of abstract integral in Riesz spaces, Rend. Circ. Matematico di Palermo, Serie II, 46 (1997), 255-278.

[16] D. Candeloro - A. Martellotti, Su alcuni problemi relativi a misure scalari subadditive e applicazioni al caso dell'additività finita, Atti Sem. Mat. Fis. Univ. Modena, 27, (1978), 284-296.

[17] D. Candeloro - A. Martellotti, Sul rango di una massa vettoriale, Atti Sem. Mat. Fis. Univ. Modena, 28, (1979), 102-111.

[18] D. Candeloro - A. R. Sambucini, Order-type Henstock and Mc Shane integrals in Banach lattices setting, arXiv:1401.7818 [math.FA], Sisy 20014- IEEE 12th International Symposium on Intelligent Systems and Informatics, Subotica - Serbia; 09/2014

[19] D. Candeloro - A.R. Sambucini, Comparison between some norm and order gauge integrals in Banach lattices, PanAmerican Mathematical Journal, 25 (3), (2015), 1-16.

[20] G. Choquet, Theory of capacities; Ann. Inst. Fourier, 5 (1953), 131-295. 
[21] G. Coletti - G. Regoli, Sulla funzione di distribuzione di una misura di probabilità finitamente additiva, Rend. Mat. Appl. 1 (7), (1981), 319-329.

[22] G. Coletti - B. Vantaggi, Conditional not-additive measures and fuzzy sets, in: Proc. ISIPTA 2013, pp. 67-76.

[23] G. Coletti - D. Petturiti - B. Vantaggi, Choquet expected utility representation of preferences on generalized lotteries, Information processing and management of uncertainty in knowledge-based systems. Part II, 444-453, Commun. Comput. Inf. Sci., 443, Springer, (2014).

[24] O. Delgado - E.A. Sánchez Pérez, Choquet type $L^{1}$-spaces of a vector capacity, Fuzzy Sets and Systems 327 (2017), 98-122.

[25] D. Denneberg, Non additive measures and integrals, Kluwer Acad. Publ. Ser. B Vol. 27, (1994).

[26] L. Di Piazza - V. Marraffa, Pettis integrability of fuzzy mappings with values in arbitrary Banach spaces, Math. Slov 66 (5), , (2016) 1119-1138, arXiv:1710.04124 1.

[27] E. Hewitt - K. Stromberg, Real and abstract analysis, Springer-Verlag Berlin (1969).

[28] W. Hildebrand, Core and Equilibria of a Large Economy, Princeton University Press, Princeton, (1974).

[29] L.C. Jang - B. M. Kil - J. S. Kwon, Some properties of Choquet integrals of set-valued functions, Fuzzy Sets and Systems, 91, (1997), 95-98.

[30] L.C. Jang - J. S. Kwon, On the representation of Choquet integrals of set valued functions and null sets, Fuzzy Sets and Systems, 112, (2000), 233-239.

[31] S. J. Leese, Multifunctions of Souslin type, Bull. Austr. Math. Soc., 11, (1974), 395-411.

[32] J. Li - R. Mesiar - E. Pap, The Choquet integral as Lebesgue integral and related inequalities, Kybernetika, 46 (6), (2010), 198-1107.

[33] J. Li - R. Mesiar - E. Pap, Atoms of weakly null-additive monotone measures and integrals, Information Sciences 257, (2014), 183-192.

[34] R. Mesiar, Choquet-like integrals, J. Math. Anal. Appl. 194, (1995), 477-488.

[35] R. Mesiar - J. Šipoš, A theory of fuzzy measures: integration and its additivity, International Journal of General Systems, 23 (1),(2007), 49-57, Doi: 10.1080/03081079408908029

[36] R. Mesiar - A. Kolesárová - H. Bustince -G.P. Dimuro - B.C. Bedregal, Fusion functions based discrete Choquetlike integrals, European J. Oper. Res. 252 (2), (2016), 601-609.

[37] T. Murofushi - M. Sugeno, A Theory of Fuzzy measures: Representations, the Choquet Integral and Null Sets, J. Math. Anal. and Appl., 159, (1991), 532-549.

[38] L. Nachbin, Topology and order, Van Nostrand (1965).

[39] Y. Narukawa - V. Torra, Fuzzy measures and Choquet integral on discrete spaces, Computational Intelligence, Theory and Applications, Volume 33 of the series Advances in Soft Computing pp. 573-581 (2005).

[40] E. Pap, Pseudo-additive measures and their applications, Handbook of Measure Theory, E. Pap ed., Elsevier (2002)

[41] A.R. Sambucini, The Choquet integral with respect to fuzzy measures and applications, to appear in Math. Slov. 67 6, (2017) Doi: 10.15151/ms-2017-0049

[42] C. Stamate - A. Croitoru Non linear integrals, properties and relationships, Recent Advances in Telecommunications, Signal and Systems, ISBN: 978-1-61804-169-2, WSEAS Press, 118-123

[43] K. Vind, Edgeworth Allocations in Exchange Economy with many Traders, Int. Econom. Review, 5,(1964), 165177.

[44] J. Zhang, Subjective ambiguity, expected utility and Choquet expected utility, Economic Theory 20, (2002), 159181. 